\title{
Integration of Research, Public Health, and Hospital Interventions as a Successful Model for Controlling COVID-19 Pandemic: A Perspective
}

\author{
Mohammad Hassan Emamian ${ }^{1}$, Reza Chaman ${ }^{2}$, Ahmad Khosravi², Hossein Sheibani ${ }^{3}$, \\ Ehsan Binesh ${ }^{3}$, Hamid Vahedi ${ }^{3}$, Hamid Reza Khajeha ${ }^{1}$, Fariba Zare ${ }^{4}$, Mohammad Saeidi ${ }^{1}$, \\ and Marzieh Rohani-Rasaf ${ }^{2}$ \\ ${ }^{1}$ Ophthalmic Epidemiology Research Center, Shahroud University of Medical Sciences, \\ Shahroud, Iran. \\ ${ }^{2}$ Department of Epidemiology, School of Public Health, Shahroud University of Medical \\ Sciences, Shahroud, Iran \\ ${ }^{3}$ Clinical Research Development Unit, Imam Hossein Hospital, Shahroud University of \\ Medical Sciences, Shahroud, Iran \\ ${ }^{4}$ Center for Health Related Social and Behavioral Sciences Research, Shahroud University \\ of Medical Sciences, Shahroud, Iran
}

June 15,2020

\begin{abstract}
The COVID-19 pandemic has been a serious health problem in most countries in the last few months, with every country adopting different preventive and therapeutic measures based on their specific circumstances. The epidemic began in Iran on February 19, 2020 and gradually spread across the country. The epidemic extent varies, and different preventive and therapeutic measures are taken in Iran. Shahroud and Miami Counties, covered by the Shahroud University of Medical Sciences, have experienced a highest incidence of COVID-19 in Iran. However, the epidemic is well controlled by integrating the activities of the health, treatment, and research sectors and using information technology and a proprietary software application. This model can be thus studied as a successful experience.
\end{abstract}

\section{Introduction}

Dealing with the COVID-19 is a newly-emerged global challenge in infectious diseases management. The disease first spread in China and Southeast Asia in late 2019 and gradually led to the death of a considerable number of people worldwide, especially in Europe and then the United States.${ }^{1}$ Not only has this disease posed the risk of infection-induced death, it has also brought unbearable stress. ${ }^{2}$ By May 13, 2020, there were 4,170,424 confirmed cases worldwide, and the death toll reached 287,399 people. The World Health Organization (WHO) still assesses the COVID-19 a very high-risk disease. ${ }^{1}$

Countries have responded differently to the disease. According to global recommendations, most countries have partially employed the three most important strategies (mitigation, suppression, and containment) to control the disease. ${ }^{3-6}$ Meanwhile, countries such as South Korea have been more successful in containing the epidemic, ${ }^{7}$ whereas most European countries are facing a formidable challenge. ${ }^{1,8}$

In all countries, general policies have usually been made by the ministry of health or the state for public affairs such as job closure; the instructions are then given to lower levels along with scientific medical and 
health recommendations. In the treatment sector, the medical staff (mainly doctors and nurses) are usually involved in the treatment of patients exclusively; these staff are also exposed to the risk of death. ${ }^{9-11}$ The public health sector is responsible for public education, case finding, contact tracing, and other health cares. Finally, the research sector adopts a different approach to the epidemic and takes valuable studies such as conduction of clinical trials to determine the effectiveness of new drugs and development of vaccines.

In Iran, the Ministry of Health and Medical Education benefits from structural management centralization. The ministry is mainly responsible for providing health, treatment and education services through 7 separate deputy named Health, Treatment, Education, Research and Technology, Food and Drugs, Students and cultural, and Development of Management and Resources. The Health deputy is responsible for providing primary healthcare services for the community through an extensive network of health centers across the country, whereas the Treatment deputy is in charge of coordinating and controlling the provision of services in public and private medical centers. Moreover, the Research and Technology deputy is responsible for directing and supporting health-related research. More than sixty medical universities in different provinces and regions of Iran have been assigned to provide health services. With a structure similar to that of the Health Ministry, they provide services for the regions they cover. It should be noted that integration and coordination of activities of the three deputies of Health, Treatment, and Research can provide excellent opportunities for controlling the epidemic. Fortunately, this has been implemented at Shahroud University of Medical Sciences by proposing a research-based model. This model was implemented on an approximate population of 260,000 people covered by Shahroud University of Medical Sciences (Shahroud and Miami Counties), with the highest incidence of the COVID-19 in Iran, and the epidemic has been properly controlled. ${ }^{12}$ The specifications of this model and successful experience are described below.

The University Headquarters for Prevention and Control of COVID-19 was established right after the identification of the first confirmed positive case of coronavirus in Iran on February 19, 2020. After the responsibilities were declared, each deputy took proper measures to provide adequate beds (including intensive care beds ) for the treatment of patients, supply protective equipment for healthcare workers and then for society, and provide public education. The Research and Technology deputy employed the faculty members of clinical and basic sciences and developed a pivotal research model in cooperation with the Epidemiology department. The model led to the coordination of various academic activities and their better effectiveness. The main specifications of this model are described below.

1. Designing and presenting a systematic model for the registration of data on suspected and confirmed cases of COVID-19 in hospitals and outpatient clinics including contact information, disease symptoms and their date of onset, results of CT scanning, PCR lab results, clinical examinations, prescribed drugs, vital signs, caring methods, recording cardiac monitoring, oxygen therapy in the ICU, and contact tracing.

2. Developing a proprietary software application for recording data and the access of Treatment, Health, and Research deputies so that, besides data confidentiality, patients' data related to tests, radiographies, drugs, etc. could be recorded in the system and could be entered into the system from HIS and other existing systems. In fact, rapid development of this comprehensive software application was the most important step in controlling this epidemic. Unfortunately, multiple systems are utilized, and duplicate information is sometimes registered in these systems in most cities of Iran. Data entry into these systems is very timeconsuming and due to a large amount of missing data, statistical analyses are inaccurate.

3. The Health and Treatment deputies are in contact via this system so that, immediately after hospitalization of suspected or confirmed patients, their personal, job, and contact information will be available to the system users in extensive health centers. Based on the dates and schedules defined in the system, these contacts are actively traced for up to 14 days by healthcare providers, and in addition to recording the disease symptoms, health education is provided for them. At the same time, packages of protective equipment including masks, gloves, and disinfectants are delivered to patients and their families.

4. In the active contact tracing program, the necessary tests and treatment are performed on symptomatic individuals in contact with the patient, and if necessary, they are referred to hospitals via the same system. 
In this program, in addition to healthcare providers in extensive health centers, 12 supervisors monitor the health care workers activities and their records in the system. They also try to identify cases of migration or with missing contact information. The purpose of this model in the Health deputy is to target the activities and perform them accurately in a timely manner. Within near three months of the epidemic, 29,529 inperson and phone tracings were performed for 7,640 jobs and family contacts of 611 confirmed patients, a process which was very effective in controlling the epidemic.

5. Establishment of the education ward in hospitals was another important step in controlling the epidemic. In these wards, every discharged patient, along with a next of kin, receives the necessary consultations and educations for isolation up to 14 days after discharge. The patients also receive training materials, masks, and disinfectants. These trainings are also provided for the relatives of patients who die in the hospital.

6. Availability of clinical and laboratory evaluations and follow-up of the relatives, according to different levels of access, resulted in clear provision of all health and treatment staff with the patients' conditions, and honest presentation of this information led to better and more accurate cooperation of employees.

7. The management dashboard of this software application in the Health and Treatment deputies and senior managers of the university provided the managers with accurate, fast, and timely information about the epidemic for decision making.

8. Careful recording of data led clinical experts to evaluate the results of their treatment comfortably and quickly and to make the necessary changes in the treatment protocols timely. In addition, it helped the decision makers to predict the required number of beds and staff by examining the status quo and to manage regions with more cases accurately.

9. Other research projects can be performed precisely by using the accurate and systematic recorded data of this system. In this system, patients are predicted to be followed up for 90 days after discharge. In addition, data of two well-known cohort studies in Shahroud ${ }^{13,14}$ can be employed to evaluate the relationship between different exposures and the COVID-19.

10. Providing accurate and timely statistics for the society encouraged people, organizations, and NGOs to give a large volume of donation including masks and protective and required equipment. The epidemic was controlled properly due to the increased participation of people, the seriousness of health advice, closing jobs, and the staying at home instructions eventually.

11. The resultant data were employed for inter-sectoral cooperation in the first days of the epidemic before the national decisions, and all schools, universities, mosques, Friday prayers, and congregational prayers were shut down.

12. Systematic registration of data and preparation of a biobank from hospitalized patients paved the way for future studies. In other words, important investigations can be carried out in consistency to this study.

13. This program can provide data required by senior managers inside and outside of the Ministry of Health easily and accurately. Certainly, these statistics are highly effective in making the right decisions.

14. Requesting the PCR test and its simple registration in all covered centers, sending the sample quickly for testing, recording the results in the system, preparing outputs to complete the data of other systems, and exact registration of patients in the form of a registration program and even a cohort study were among the other advantages of the system and the joint action.

15. This system is an instrument for determining clinical severity, transmission rate, and optimization of treatment options. Recording and evaluating the symptoms in the relatives of patients gradually determine changes in the symptoms of patients and sensitization of the health staff to various symptoms.

16. The use of this system and its information can actually equip the treatment sector and dedicate the largest and the best equipped hospital in Shahroud exclusively for COVID-19 patients with the presence of the most experienced specialized workers. In addition to providing the non-COVID-19 patients with 
therapeutic requirements, this decision minimized the risk of staff infection and prevented contamination of other hospitals. Thanks to its physical size and structure as well as efficient ventilation facilities, all of which were led to the optimal and economic use of facilities.

\section{Conclusion}

Obviously, close and continuous monitoring is necessary for accurate tracing and prediction of the COVID-19. Decisions made by officials at the time of crisis should be culturally and socially appropriate and sensitive. Timely, accurate, and transparent information on the epidemic in society and workplace accelerates the implementation of virus-controlling strategies. The University Headquarters for Prevention and Control of the COVID-19 in Shahroud quickly recognized the crisis and activated the emergency management structures to prevent the disease spread.

This comprehensive research project was started in cooperation with three deputies of Health, Treatment, and Research aiming to trace the contacts of the confirmed cases as soon as possible. It was then continued by slowing spread of the epidemic through early detection of the confirmed cases. In contrast to very fast transmission of SARS-CoV-2, manual tracing aiming to prevent the spread of epidemics is very slow; ${ }^{15}$ therefore, information-based technologies are required to trace individuals in contact with the confirmed cases quickly and effectively, to give immediate notification, and to start isolation. Each patient with the COVID-19 should be detected, treated, educated and isolated immediately, and their contacts should be identified effectively as well. All of these steps were implemented properly in this program. Moreover, by developing a comprehensive and complete structure and using the opinions of experts in each deputy as well as feedback received from people, this program further improved in accordance with the daily needs of the region.

Considering specific individual, cultural, and social characteristics of each city, it is important that every region should assess its own risks and take prompt actions on an appropriate scale to reduce both the COVID-19 transmission and its economic, general, and social impacts. Coordination and joint actions of Health, Treatment, and Research sectors and the use of information technology according to local conditions can serve as a successful model for controlling epidemics.

Acknowledgements: This work was supported by Shahroud University of Medical Sciences (Grant No. 98126).

Conflicting Interests: The Authors declare that there is no conflict of interest.

\section{References}

1. World Health Organization, Coronavirus disease (COVID-2019) situation report-114. . 13 May 2020, https://www.who.int/docs/default-source/coronaviruse/situation-reports/20200513-covid-19sitrep-114.pdf?sfvrsn=17ebbbe_4.

2. Xiao C. A Novel Approach of Consultation on 2019 Novel Coronavirus (COVID-19)-Related Psychological and Mental Problems: Structured Letter Therapy. Psychiatry investigation. 2020;17(2):175-6.

3. Walensky RP, Del Rio C. From Mitigation to Containment of the COVID-19 Pandemic: Putting the SARS-CoV-2 Genie Back in the Bottle. JAMA. 2020.

4. Walker PG, Whittaker C, Watson O, Baguelin M, Ainslie KEC, Bhatia S. The Global Impact of COVID-19 and Strategies for Mitigation and Suppression. Imperial College London. 26 March 2020.

5. Preparedness and mitigation measures for COVID-19: Update No 9. 17-20 April 2020.

6. World Health Organization, Critical preparedness, readiness and response actions for COVID-19: interim guidance. 22 March 2020.

7. Korea Centers for Disease Control and Prevention. April 11, 2020 [Available from: https://www.cdc.go.kr/board/board.es?mid=a30402000000\&bid=0030. 
8. New York Times. Coronavirus in the U.S.: latest map and case count. April 29, 2020. Accessed April 29, 2020. https://www.nytimes.com/interactive/2020/us/coronavirus-us-cases.html [

9. Chang D, Xu H, Rebaza A, Sharma L, Cruz CSD. Protecting health-care workers from subclinical coronavirus infection. The Lancet Respiratory Medicine. 2020;8(3):e13.

10. Casanova LM, Rutala WA, Weber DJ, Sobsey MD. Effect of single-versus double-gloving on virus transfer to health care workers' skin and clothing during removal of personal protective equipment. American journal of infection control. 2012;40(4):369-74.

11. Tran K, Cimon K, Severn M, Pessoa-Silva CL, Conly J. Aerosol generating procedures and risk of transmission of acute respiratory infections to healthcare workers: a systematic review. PloS one. 2012;7(4).

12. Khosravi A, Chaman R, Rohani-Rasaf M, Zare F, Mehravaran S, Emamian MH. The basic reproduction number and prediction of the epidemic size of the novel coronavirus (COVID-19) in Shahroud, Iran. Iran (3/25/2020). 2020.

13. Fotouhi A, Hashemi H, Shariati M, Emamian MH, Yazdani K, Jafarzadehpur E, et al. Cohort profile: Shahroud Eye Cohort Study. Int J Epidemiol. 2013;42(5):1300-8.

14. Emamian MH, Hashemi H, Khabazkhoob M, Malihi S, Fotouhi A. Cohort Profile: Shahroud Schoolchildren Eye Cohort Study (SSCECS). Int J Epidemiol. 2019;48(1):27-f.

15. Ferretti L, Wymant C, Kendall M, Zhao L, Nurtay A, Bonsall DG, et al. Quantifying dynamics of SARS-CoV-2 transmission suggests that epidemic control and avoidance is feasible through instantaneous digital contact tracing. medRxiv. 2020. 